\title{
ESTIMATIVA DA IRRADIAÇÃO TOTAL SOBRE UMA SUPERFÍCIE INCLINADA A PARTIR DA IRRADIAÇÃO GLOBAL NA HORIZONTAL
}

\author{
José Scolar, 'Dinival Martins, ${ }^{2}$ João Francisco Escobedo ${ }^{3}$ \\ Recebido em 21 out. 2003 / Aceito em 22 jun. 2004 \\ Received oct. 21, 2003 / Accepted jun. 22, 2004
}

\section{ABSTRACT}

A modeling study of the total irradiation reaching a tilted surface facing towards the Equator at a place with the same latitude $\left(-22.85^{\circ}\right)$, using the Daily partitioning energy, is presented. In this study three total irradiation incident on tilted surface estimate models (LIU; JORDAN, 1963; HAY, 1979; PEREZ et al., 1987) were modified in two different manners, to improve irradiation estimative model for tilted surface knowing only the global irradiation. The first modification consisted to apply empirical functions to determine the diffuse irradiation by the global radiation measurements. These empirical functions were tuned using a linear regression technique, the fraction of the diffuse irradiation $\left(K_{d}\right)$ from the global irradiation, was correlated with the clearness index $\left(K_{d}\right)$. In this case the highest correlation found was a fourth order polynomial function. In the second modification the fraction of the direct irradiation $K_{B H}$ from the global irradiation was correlated assuming a linear correlation at given intervals of $K_{t}$. Model results showed that the empirical relationship $K_{B H}=f\left(K_{t}\right)$ presented better results and the Hay model presented a better performance compared with the two others models for the Botucatu region.

Keywords: Solar irradiation, Tilted surface, models.

\section{RESUMO}

Neste trabalho realizou-se um estudo de modelagem da irradiação total que incide sobre uma superfície inclinada, com face voltada para 0 Equador e inclinação de 22,85', igual a da latitude local, usando partição diária de energia em Botucatu - SP. Dos vários modelos de estimativa da irradiação total em uma superfície inclinada existentes na literatura, três deles (LIU; JORDAN, 1963; HAY, 1979, PEREZ et al., 1987) foram utilizados e modificados de duas maneiras diferentes, com o propósito de se obter um modelo que utilize somente a irradiação global na estimativa da irradiação total na superfície inclinada. Na primeira, a irradiação difusa foi estimada em função da irradiação global, através do ajuste empírico utilizando a técnica de regressão linear, na forma $K_{d}=f\left(K_{t}\right)$, fração da difusa contida na global $\left(K_{d}\right)$, com 0 índice de claridade $\left(K_{t}\right)$. Nesse caso, o melhor ajuste é dado por um polinômio de quarto grau. Na segunda modificaçãa, a irradiação direta $K_{B H^{\prime}}$ fraçã̃o da irradiação direta contida na irradiação global, foi ajustada através da técnica de regressão linear, em função do índice de claridade, na forma $K_{B H}=f\left(K_{t}\right)$. Nesse caso, o melhor ajuste foi linear, obtido para intervalos específicos de $K_{t}$. Os resultados obtidos com essas modificações mostram que os modelos têm melhores desempenhos para a modificação feita com a função $K_{B H}=f\left(K_{t}\right)$, e 0 modelo modificado de Hay apresenta 0 melhor desempenho na estimativa da irradiação total em Botucatu-SP.

Palavras-chave: Irradiação solar, Superfície inclinada, modelos.

\footnotetext{
1 Pesquisador aposentado do Instituto de Pesquisas Meteorológicas da UNESP de BAURU - Rua Martim Afonso 11-28 Vila Souto - CEP 17051-040 Bauru, SP, Brasil Fone (14) 32272832 E-mail: scolar@uol.com.br

2 Faculdade de Ciências Agronômicas - Departamento de Ciências Ambientais da UNESP- Botucatu - SP - Fazenda Experimental Lageado - CX Postal 237 CEP 18603-970 Botucatu, SP, Brasil. Fone (14) 38117162 - E-mail: Dinival@fca.unesp.br

3 Faculdade de Ciências Agronômicas - Departamento de Ciências Ambientais da UNESP- Botucatu - SP - Fazenda Experimental Lageado - Cx Postal 237 CEP $18603-970$ Botucatu, SP, Brasil. Fone (14) 38117162 - E-mail: Escobedo@fca.unesp.br
} 


\section{INTRODUÇÃOO}

A crescente demanda por informações sobre a utilização da radiação solar como fonte suplementar e alternativa às fontes convencionais de energia, requer o conhecimento detalhado das características climáticas da radiação solar incidente sobre superfícies inclinadas em relação à superfície horizontal, para serem usadas em uma grande variedade de aplicações, incluindo projetos de engenharia para coletores solares, projetos de arquitetura, planejamento urbano, estudos agronômicos de insolação sobre vegetação e em estudos micrometeorológico sobre circulação local. Para satisfazer qualquer um desses estudos é necessário conhecer a intensidade da radiação solar que incide sobre uma superfície inclinada e sua variação sazonal por um período mínimo de um ano. Contudo, o alto custo instrumental e a necessidade de pessoal operacional dificultam a implantação e operação de uma rede de estações de radiometria solar. 0 resultado é um número limitado de estações ao redor do globo terrestre para observações das componentes da radiação solar na superfície horizontal e principalmente a observação da radiaçãa solar em uma superfície inclinada, já que nem todas as estações de radiometria solar fazem essas medidas.

Por causa da falta de observações da radiação solar que incide em uma superfície inclinada, vários modelos teóricos de estimativa foram desenvolvidos, utilizando medidas da radiação global e da componente direta da radiação obtidas na superfície horizontal (LIU; JORDAN, 1963; HAY, 1979; TEMPS; COULSON, 1977; KLUCHER, 1979, PEREZ et al., 1987). Esses modelos utilizam individualmente os valores da radiação direta, difusa e da refletida pela superfície do solo como componentes da radiação total que incide sobre uma superfície inclinada. A única diferença entre os vários modelos existentes está no tratamento da radiação difusa que incide sobre a superfície inclinada, devido ao seu comportamento anisotrópico.

Em muitas localidades a componente direta da radiação solar não é medida, existindo somente observações da radiação global na superfície horizontal. Nessa circunstância, para estimar a radiação total que incide sobre uma superfície inclinada, as componentes difusa e direta da radiação solar devem ser estimadas, e isto pode ser feito usando modelos conceituais que relacionam a fração da radiação difusa contida na radiação global com o índice de claridade, fração entre as irradiações global e do topo da atmosfera, $K_{d}=f\left(K_{t}\right)$, ou a fração da direta contida na global com 0 índice de claridade $K_{B H}=f\left(K_{t}\right)$. São exemplos dessas correlaçães os modelos de Erbs Klein e Duffie (1982); Oliveira e outros (2002); Ricieri (1988), Gueymard (1993); Fevermann e Zemel (1992). Nesse caso, são utilizados para estimar a radiação total na superfície os modelos empíricos de estimativa da radiação difusa ou da direta, desenvolvidos em uma determinada localidade, em conjunto com os valores da radiação solar global medida localmente na superfície horizontal inclinada (KLEIN, 1977; BUGLER, 1977; NOTTON, MUSELLI; LOUCHE, 1996; BEHR, 1997).

0 objetivo deste trabalho é obter um modelo para estimar a irradiação total diária que incide em uma superfície inclinada para Botucatu-SP, usando somente a radiação global medida na superfície horizontal, utilizando modelos modificados de estimativa da radiação total incidente na superfície inclinada, com as funçōes $K_{d}=f\left(K_{t}\right)$, e por $K_{B H}=f\left(K_{t}\right)$, e verificar o desempenho dos modelos modificados por essas relações empíricas.

\section{CONJUNTO DE DADOS}

Os dados das irradiâncias solar direta e global para a superfície horizontal e da total em um plano inclinado igual a latitude local (22 $54^{\prime}$ ) e a face voltada para 0 Equador, foram coletados na Estação de Radiometria Solar da UNESP de Botucatu-SP (latitude 22 $51^{\prime}$ Sul, longitude $48^{\circ} 26^{\prime}$ Oeste, altitude $786 \mathrm{~m}$ ), entre setembro de 1997 e agosto de 2001. As leituras foram feitas a cada segundo e as médias armazenadas a cada cinco minutos. Cerca de $9 \%$ dos dados foram desprezados na análise de consistência. Os modelos (LIU; JORDAN, 1963; HAY, 1979; PEREZ et al., 1987) de estimativa da irradiação total na superfície inclinada foram avaliados, e os modelos modificados, desenvolvidos com os dados coletados no período de setembro de 1997 a agosto de 2000 (1034 dias), validados com os dados obtidos no período setembro de 2000 a agosto de 2001 (332 dias).

A irradiância direta na incidência normal foi obtida por meio de um pireliômetro EPPLEY-NIP, acoplado a um rastreador solar EPPLEY modelo ST-3, fixo na direção norte-sul geográfica e ajustado na latitude local. A irradiância global no plano horizontal foi obtida com auxilio de um piranômetro EPPLEY-PSP, posicionado num plano horizontal e, no plano inclinado foi obtida também com auxilio de um piranômetro EPPLEY-PSP, posicionado paralelamente sobre o plano inclinado.

As irradiações diárias $\left(H_{0}^{d}, H_{\beta 0}^{d}, H_{b}^{d}, H_{g}^{d}, H_{d}^{d}\right.$, $H_{\beta T O}^{d}$ ), no topo da atmosfera para a superfície horizontal, no topo da atmosfera para a superfície inclinada, direta projetada na horizontal, global no plano horizontal, difusa na superfície horizontal obtida pela diferença entre as irradiações global e direta na horizontal e total observada na superfície inclinada, foram obtidas através da integração das respectivas curvas de irradiância, integradas do nascer ao pôr-do-sol. 


\section{MODELOS DE ESTIMATIVA DA IRRADIAÇÃO TOTAL}

A irradiação total diária $H_{\beta T}^{d}$, que incide em uma superfície inclinada, com um ângulo $\beta$ de inclinação em relação a superfície horizontal, é dada pela soma das irradiações direta $H_{\beta b}^{d}$, difusa $H_{\beta d}^{d}$ e refletida pelo solo $H_{\beta}^{d}$, isto é:

$H_{\beta T}^{d}=H_{\beta b}^{d}+H_{\beta d}^{d}+H_{\beta r}^{d}$

A irradiação direta que atinge a superfície inclinada, segundo Iqbal (1983), é calculada através de correção da irradiação direta projetada na horizontal $H_{b h}^{d}$, dada pela seguinte expressão:

$$
H_{\beta b}^{d}=H_{b h}^{d} R_{B}
$$

onde, $R_{B}=\cos \left(\theta_{\beta}\right) / \cos \left(\theta_{z}\right)$ é o fator de correçã̃o devido à mudança no ângulo de incidência dos raios solares sobre a superfície inclinada, $\theta_{z}$ é 0 ângulo zenital do Sol e $\theta_{\beta}$ é 0 ângulo de incidência dos raios solares sobre um superfície inclinada com a face voltada para 0 Equador.

Considerando-se que a reflexão pela superfície do solo seja isotrópica, a fração da energia refletida pelo solo que atinge a superfície inclinada é dada por:

$$
H_{\beta r}^{d}=H_{g}^{d} \rho \frac{1}{2}(1-\cos (\beta))
$$

onde, $\rho$ é 0 albedo da superfície definido como a irradiação refletida por uma superfície sobre a irradiação incidente nessa superfície, $\beta$ é o ângulo de inclinação e o termo $\left[\frac{1}{2}(1-\cos (\beta))\right]$ é a razão entre a irradiação incidente na superfície inclinada sobre a radiação refletida pelo solo.

Esse tratamento matemático para as irradiações direta e refletida pelo solo, que incidem numa superfície inclinada, é comum à maioria dos modelos, excetuando-se uns poucos, como os de Temps e Coulson (1977) e Gueymard (1987), que dão tratamento anisotrópico para a irradiação refletida pelo solo. A diferença básica entre os vários modelos de estimativa da irradiação total, está na modelagem da irradiação difusa, desde que a sua distribuição espacial e temporal geralmente é desconhecida devido ao seu comportamento anisotrópico, tornando-a uma variável difíil de ser modelada.

\section{Modelos de estimativa da irradiação difusa}

Normalmente são utilizadas três subcomponentes para avaliar 0 comportamento anisotrópico da radiação difusa que incide na superfície inclinada: circunsolar, brilho horizontal e radiação difusa isotrópica. A irradiação difusa que vem da região ao redor do disco solar é chamada de circunsolar, sendo predominantemente resultante do espalhamento devido aos aerossóis existente na atmosfera. 0 aumento na intensidade da radiação difusa próximo ao horizonte é chamado de brilho horizontal, em virtude de a radiação incidente percorrer uma trajetória maior na atmosfera próximo ao horizonte e também por causa das múltiplas reflexões na atmosfera terrestre. Isotrópica é o restante da radiação difusa uniformemente distribuída na porção restante da hemisfério celeste.

Vários modelos têm sido propostos para estimar a irradiação difusa que atinge uma superfície inclinada; no entanto, nem todos consideram essas três subcomponentes. Desses vários modelos existentes na literatura apenas três deles foram selecionados e utilizados para estimar a irradiação difusa, (LIU; JORDAN, 1963; HAY, 1979; PEREZ et al., 1987).

Liv e Jordan (1963) assumem que a intensidade da irradiação difusa é uniforme em todo o hemisfério celeste. Sendo portanto, válida para condições de céu nublado, condição na qual a irradiação difusa é suposta isotrópica. Seguindo o mesmo procedimento adotado para a irradiação refletida pelo solo, Liv e Jordan (1963) propõem a seguinte equação para o cálculo da irradiação difusa que atinge uma superfície inclinada:

$$
H_{\beta d}^{d}=H_{d}^{d} \frac{1}{2}(1+\cos \beta)
$$

onde 0 termo $\left[\frac{1}{2}(1-\cos (\beta))\right]$ é a raz̃ão entre a irradiação difusa incidente na superfície inclinada sobre a irradiação difusa vinda da cúpula do céu.

Esse modelo apresenta bons resultados para condições de céu nublado, quando a irradiação difusa assume comportamento isotrópico. № entanto, em condições de céu claro, a irradiação difusa tem um comportamento anisotrópico e o modelo subestima os valores calculados. Portanto, para o cálculo da irradiação difusa que atinge uma superfície inclinada, é necessário que os modelos incorporem os efeitos tanto da isotropia quanto da anisotropia da irradiação difusa.

Hay (1979) considera a irradiação difusa composta das subcomponentes circunsolar vinda diretamente do disco solar e a isotropicamente distribuída vinda do resto do hemisfério celeste. Para cada componente é dado um peso específico, através do índice anisotrópico definido como a razão entre a irradiaçã̃o direta na superfície horizontal e a irradiação extraterrestre: $\left(A_{I}=H_{b h}^{d} / H_{0}^{d}\right)$. Este índice define a percentagem da irradiação difusa a ser tratada como circunsolar, com a percentagem restante considerada isotrópica. Em condições de céu claro, o índice anisotrópico tende a um, e a irradiação difusa circunsolar é mais fortemente ponderada do que a irradiação difusa 
isotrópica. Em condições de céu nublado, o índice anisotrópico tende a zero e toda a irradiação difusa é tratada como isotrópica. Esse comportamento é consistente com a irradiação difusa observada. A irradiação difusa circunsolar ponderada através do índice anisotrópico é projetada na superfície inclinada da mesma maneira que a componente da irradiação direta, sendo a porcentagem restante tratada como isotrópica. Portanto, a irradiação difusa que atinge o plano inclinado, no modelo proposto por Hay (1979) é dada pela seguinte equação:

$$
H_{\beta d}^{d}=H_{d}^{d}\left[A_{I} R_{B}+\frac{1}{2}(1+\cos \beta)\left(1-A_{I}\right)\right]
$$

Utilizando o mesmo desenvolvimento de Hay (1979), Perez e outros (1987) consideram a irradiação difusa sendo composta pela soma das componentes isotrópica, circunsolar e o brilho horizontal. Na versão mais simples do modelo (PEREZ et al., 1987), válida para superfícies com face inclinada para o Equador, a irradiação difusa circunsolar é considerada originar-se em um ponto no centro do disco solar. Toda energia decorrente do brilho horizontal origina-se na região infinitesimal de $\left(0^{\circ}\right)$ de elevação. Assim, a expressão de Perez e outros (1987) é escrita da seguinte maneira:

$$
H_{\beta d}^{d}=H_{d}^{d}\left[\frac{1}{2}(1+\cos \beta)(1-F)_{1}+F_{1} R_{b}+F_{2}(\operatorname{sen} \beta)\right]
$$

Onde, $F_{1}$ representa a contribuição da irradiação difusa circunsolar e $F_{2}$ é a contribuição da irradiação difusa horizontal. Esses dois parâmetros são considerados independentes um do outro e são determinados empiricamente através de dados observacionais.

\section{MODELOS MODIFICADOS DE ESTIMATIVA DA IRRADIAÇÃO TOTAL}

As medidas de irradiação global na superfície horizontal, em valores horários ou diários, são feitas em várias localidades, mas o mesmo não ocorre para as medidas de irradiação total em superfícies inclinadas. No entanto, avaliações de irradiação total em superfícies inclinadas são de fundamental importância nas estimativa da radiação absorvida por superfícies topográficas com inclinação natural, ou superfícies com inclinação forçada (caso de aquecedores solares).

Com a finalidade de estimar a irradiação incidente sobre superfícies inclinadas com a face voltada para o Equador, é testado um modelo matemático simples de estimativa que utiliza apenas a irradiação solar global observada na superfície horizontal em conjunto com modelos conceituais de estimativa das irradiações difusa e direta.
Utilizando as equações (2, 3 e 4), o modelo isotrópico de Liv e Jordan (1963) para a estimativa da irradiação total em uma superfície inclinada é escrito da seguinte maneira:

$$
\begin{aligned}
& H_{\beta T}^{d}=\left(H_{g}^{d}-H_{d}^{d}\right) R_{B}{ }^{+} H_{g}^{d} \rho \frac{1}{2}(1-\cos \beta)^{+} \\
& +H_{d}^{d} \frac{1}{2}(1+\cos \beta)
\end{aligned}
$$

Como sugerido por Klein (1977), dividindo a equação (7) pela irradiação global medida na horizontal ( $\left.H_{g}^{d}\right)$, a Equaçã̃o (7) pode ser escrita da seguinte maneira:

$H_{\beta T}^{d}=H_{g}^{d}\left[\left(1-K_{d}\right) R_{B}+\rho \frac{1}{2}(1-\cos \beta)+K_{d} \frac{1}{2}(1+\cos \beta)\right]$

onde $K_{d}=\frac{H_{d}^{d}}{H_{d}^{d}}$, é a razão entre as irradiações difusa e global na superfície horizontal.

A Equação (7) também pode ser escrita em função da fração da irradiação direta contida na irradiação global da seguinte maneira:

$$
\begin{aligned}
& H_{\beta T}^{d}=H_{g}^{d}\left[K_{B K} R_{B}+H_{g}^{d} \rho \frac{1}{2}(1-\cos \beta)+\right. \\
& \left.H_{g}^{d}\left(1-K_{B K}\right) \frac{1}{2}(1-\cos \beta)\right]
\end{aligned}
$$

onde, $K_{B H}=\frac{H_{b}^{d}}{H_{g}^{d}}$, é a razão entre as irradiações direta projetada na superfície horizontal e a global na superfície horizontal.

0 mesmo procedimento foi adotado para os modelos de Hay (1979) e Perez e outros (1987).

\section{RESULTADOS E DISCUSSÃO}

\section{Modelos conceituais de estimativa das irradiações difusa e direta}

Os modelos modificados de estimativa da irradiação total em uma superfície inclinada exigem o conhecimento da irradiação global medida na superfície horizontal e da irradiação difusa na horizontal estimada através de modelos conceituais que correlacionam a componente difusa, através da fração difusa $\left(K_{d}\right)$, com a irradiação global através do índice de claridade $K_{t}=H_{g}^{d} / H_{0}^{d}$, razão entre as irradiações global e no topo da atmosfera, isto é, $K_{d}=f\left(K_{t}\right)$, ou o conhecimento da irradiação direta na horizontal estimada através de modelos conceituais que correlacionam a componente direta, através da fração 
direta $\left(K_{B H}\right)$, com a irradiação global através do índice de claridade $K_{t^{\prime}}$ isto é, $K_{B H}=f\left(K_{t}\right)$.

Os modelos conceituais de estimativa das irradiações difusa e direta baseados no índice de claridade, são chamados do tipo liv e Jordan (1960), a partir do momento em que esses autores foram os primeiros a propor tal correlação. Esse tipo de correlação é encontrado em diversos trabalhos para diferentes localidades (KLEIN, 1976; ERBS; KLEIN; DUFFIE., 1982; GUEYMARD, 1993; RICIERI, 1998; OLIVEIRA et al., 2002). No desenvolvimento dos modelos conceituais de estimativa da irradiação difusa e direta, foram utilizados os dados coletados de setembro de 1997 a agosto de 2000, totalizando 1034 valores que passaram no teste de consistência.

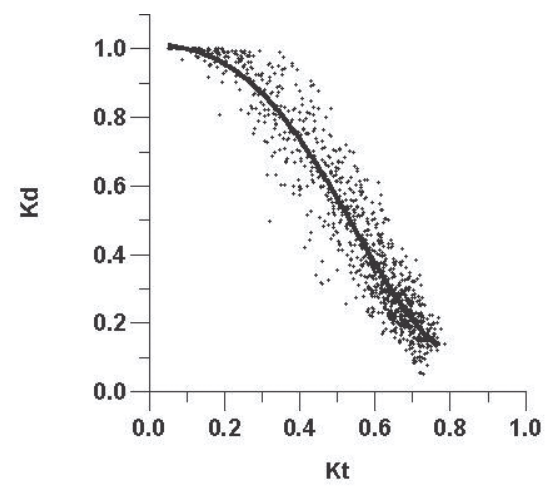

Figura 1 - Distribuição diária da irradiação difusa $\left(K_{d}\right)$ em função da irradiação global $(K)$, e o modelo ajustado por regressão polinomial de $4^{0}$ grau (linha contínua).

Figure 1 - Daily distribution of diffuse irradiation $\left(K_{d}\right)$ as a function of global irradiation $(K)$, and the fourth order polynomial fitted model (full line).

A Figura 1 mostra os valores diários de $K_{d^{\prime}}$ em função de $K_{t^{\prime}}$ e a curva ajustada por regressão linear múltipla. Nota-se que quando $K_{d}$ tende a um, $K_{t}$ tende a zero, e quando $K_{d}$ tende a zero, $K_{t}$ tende a um. No intervalo intermediário, a correlação entre essas duas variáveis pode assumir tendência linear, exponencial, ou polinomial, utilizando polinômios de diferentes graus. Dessa maneira, alguns autores correlacionam $K_{d}=f\left(K_{t}\right)$, em função de intervalos específicos de $K_{t}$.

Oliveira e outros (2002), trabalhando com dados coletados na cidade de São Paulo, concluem que o melhor ajuste é obtido com um polinômio de quarto grau, impondo limites máximos e mínimos para $K_{t}$.

Ricieri (1998) fez extensiva revisão dos modelos conceituais que correlacionam essas duas variáveis e concluiu que quando é considerada a partição diária, das irradiações global, difusa e no topo da atmosfera, a regressão linear múltipla polinomial de quarto grau fornece o melhor ajuste para $K_{d}$ em função de $K_{t}$, para Botucatu-SP.

A equação de regressão linear múltipla obtida com valores diários para Botucatu-SP, no ajuste da curva polinomial de $K_{d}$ em função de $K_{t^{\prime}}$ de quarto grau é dada pela Equação (10), com o coeficiente de determinação, $R^{2}=0,91$.

$K_{d}=0,993+0,178 K_{t}-0,945 K_{t}^{2}-4,71 K_{t}^{3}+4,89 K_{t}^{4}$

A Figura 2 mostra os valores diários de $K_{B H^{\prime}}$ em função de $K_{t}$ e a curva ajustada por regressão linear. Nota-se que, para pequenos valores de $K_{t} K_{B H}$ tende a zero, e quando $K_{B H}$ tende a um, $K_{t}$ também tende a um. Portanto, acima de um determinado valor de $K_{t}$ existe linearidade entre as duas variáveis, sugerindo que a correlação $K_{B H}=$ $f(K)$, deva ser feita em função de intervalos específicos de $K_{i}$. Nesse caso, $K_{B H}=0$ para valores de $K_{t} \leq 0,25$, condições de céu nublado. Para valores de $K_{t}>0,25$ a correlação entre essas duas variáveis assume tendência linear.

A equação de regressão linear obtida com valores diários para Botucatu-SP, no ajuste da curva polinomial de $K_{B H}$ em função de $K_{t^{\prime}}$ é dada pela Equação (11), com o coeficiente de determinação, $R^{2}=0,90$.

$$
K_{B H}=-0,386+1,572 K_{t} \text {, para } K_{t}>0,25
$$

$K_{B H}=0$, para $K_{t} \leq 0,25$

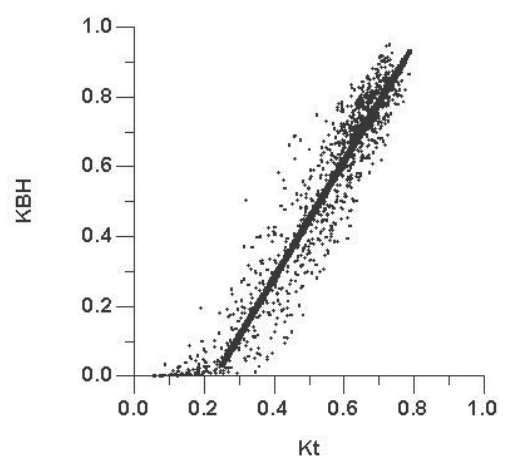

Figura 2 - Distribuição diária da irradiação direta $\left(K_{B H}\right)$ em função da irradiação global $(K)$ e o modelo ajustado por regressão linear polinomial de $1^{0}$ grau (linha contínua).

Figure 2 - Daily distribution of direct irradiation $\left(K_{B H}\right)$ as a function of global irradiation $\left(K_{t}\right)$, and the first order polynomial fitted model (full line). 


\section{Avaliação dos modelos de estimativa da irradiação total na superfície inclinada}

Nos modelos modificados de estimativa da irradiação total diária através $K_{d}=f\left(K_{t}\right)$ e por $K_{B H}=f\left(K_{t}\right)$, o procedimento adotado foi substituir, nos modelos de Liv e Jordan (1963), Hay (1979) e Perez e outros (1987), os valores observados da irradiação global na superfície horizontal $R_{G}$ em conjunto com os valores da irradiação difusa esti- mada através da Equação (10), e, similarmente, substituir os valores observados de $R_{G}$ em conjunto com os valores da irradiação direta estimada através da Equação (11).

Na Tabela 1 estão apresentados os valores diários da Raiz Quadrada do Erro Quadrático Médio (REQM) e do Erro Médio (EM) calculados para a irradiação total estimada na superfície inclinada obtida com os vários modelos de estimativa modificados por $K_{B H}=f\left(K_{t}\right)$, $K_{d}=f\left(K_{t}\right)$ e os respectivos valores calculados para os modelos originais.

Tabela 1 - Valores de REQM e EM, para a irradiação total diária estimada com os três modelos modificados, por $K_{d}=f\left(K_{t}\right)$ e $K_{B H}=f\left(K_{t}\right)$, e os valores obtidos com os modelos originais.

Table 1 - RMSE and ME values for the daily total irradiation estimated from three modified models, by $K_{d}=f\left(K_{t}\right)$ and $K_{B H}=f\left(K_{t}\right)$, and the values obtained with the original models.

\begin{tabular}{|c|c|c|c|c|c|c|}
\hline $\begin{array}{c}\text { Modelos de estimativa da } \\
\text { irradiação total }\end{array}$ & \multicolumn{2}{|c|}{ Modelos Originais } & \multicolumn{2}{c|}{$\begin{array}{c}\text { Modelos Modificados } \\
K_{d}=f\left(K_{t}\right)\end{array}$} & \multicolumn{2}{c|}{$\begin{array}{c}\text { Modelos Modificados } \\
K_{B H}=f\left(K_{t}\right)\end{array}$} \\
\hline & REQM & EM & REQM & EM & REQM & EM \\
& $M J / m^{2}$ & $M J / m^{2}$ & $M J / m^{2}$ & $M J / m^{2}$ & $M J / m^{2}$ & $M J / m^{2}$ \\
\hline Liu e Jordan (1963) & 0,61 & $-0,38$ & 0,46 & $-0,05$ & 0,44 & $-0,05$ \\
\hline Hay (1979) & 0,36 & 0,01 & 0,47 & 0,19 & 0,46 & 0,19 \\
\hline Perez et al. (1987) & 0,38 & 0,14 & 0,51 & 0,25 & 0,47 & 0,25 \\
\hline
\end{tabular}

Na Tabela 1 verifica-se que o modelo original de Liu e Jordan (1963) tem o maior valor de dispersão $\mathrm{REQM}=0,611 \mathrm{MJ} / \mathrm{m}^{2}$ e o maior erro-médio em módulo $\mathrm{EM}=-0,38 \mathrm{MJ} / \mathrm{m}^{2}$, indicando que esse modelo subestima os valores da irradiaçãa total em uma superfície inclinada. Esse resultado é esperado porque o modelo de Liu e Jordan corrige a irradiação difusa apenas com o fator geométrico 0,5 . $(1+\cos (\beta))=0,96$, correção suficiente apenas para condições de céu nublado onde a irradiação difusa vinda do céu apresenta comportamento uniforme, ou seja isotrópico. Contudo, para condições de céu parcialmente nublado ou claro, o modelo de Liu e Jordan subestima a quantidade da irradiação difusa que incide sobre a superfície inclinada, principalmente em condições de céu claro em conseqüência de que a maior percentagem da irradiação difusa é proveniente do disco solar, ou seja a componente circunsolar. Esse resultado, demonstra claramente que 0 modelo isotrópico é deficiente na estimativa da irradiação total em uma superfície inclinada em condições de céu parcialmente nublado ou claro, principalmente por causa do comportamento anisotrópico da irradiaçã̃o difusa, tornando-se necessário que a correção seja maior do que a simples correção geométrica. 0 modelo de Hay (1979) superestima pouco os valores da irradiação total $\mathrm{EM}=0,01 \mathrm{MJ} / \mathrm{m}^{2}$ e também tem 0 menor valor de dispersão REQM $=0,36 \mathrm{MJ} / \mathrm{m}^{2}$, demostrando que a introdução do índice anisotrópico ( $A_{I}=H_{b h}^{d} / H_{0}^{d}$ ) no modelo, para caracterizar 0 estado do céu, e subdividir a irradiação difusa nas componentes circunsolar e isotrópica ponderadas por esse índice, produz excelentes resultados na estimativa da irradiação total em uma superfície inclinada. Em condições de céu claro, o índice anisotrópico tende a 1 e a componente circunsolar da irradiação difusa ponderada por esse índice tem um peso maior do que a difusa isotrópica. Em condições de céu nublado, o índice anisotrópico tende a zero e praticamente toda a irradiação difusa é tratada como isotrópica. Na formulação matemática do modelo de Perez e outros (1987), são incluídas as subcomponentes da irradiação difusa, circunsolar, isotrópica e brilho horizontal. Nota-se que de maneira geral, esse modelo superestima os valores da irradiação total na superfície inclinada e apresenta valores de dispersão comparável com o modelo de Hay (1979).

Comparando os resultados, obtidos para os modelos modificados, com os originais, verifica-se que para o modelo de Liu e Jordan (1963) e para as duas diferentes modificações feitas, existe melhoria significativa com EM e REQM, um pouco menores do que os resultados obtidos com o modelo original, embora continue subestimando os valores. Como esse modelo, na forma original, não considera condições que caracterizam 0 estado do céu, com 0 uso da irradiação difusa ou da 
direta estimada em função do índice de claridade, esta, pode ser a causa da melhoria no modelo de Liv e Jordan (1963). De maneira geral, a análise dos erros demostra que eles aumentam ligeiramente para todos os outros modelos, com pouco aumento da superestimativa e dos valores da dispersão, com similaridade para ambos os métodos de modificação quando comparados com os erros obtidos com os modelos originais, sendo que esses erros são menores para a modificação feita com a função $K_{B H}=f\left(K_{t}\right)$, provavelmente devido à correlação linear entre as duas variáveis, e pode estimar a irradiação total com razoável exatidão.

\section{VALIDAÇÃO DOS MODELOS}

Para verificar 0 comportamento dos modelos no decorrer do ano, os mesmos foram validados para um intervalo mais curto de tempo mensalmente.

A Figura 3 mostra os valores de EM obtidos mensalmente com os modelos originais de estimativa da irradiação total diária. Nota-se claramente que o modelo de Liv e Jordan (1963) subestima os valores de irradiação em praticamente todos os meses do ano, exceto janeiro e dezembro. A subestimativa, em torno de $-0,7 \mathrm{MJ} / \mathrm{m}^{2}$, é grande para os meses de outono e inverno, decorrência de que no modelo de Liv e Jordan a irradiação difusa é corrigida apenas pelo fator geométrico uma correção válida somente para irradiação difusa isotrópica. № entanto, durante 0 inverno, na região de Botucatu - SP, onde prevalecem condições de céu claro e a irradiação difusa tem caráter anisotrópico, e a correção geométrica é insuficiente, torna-se necessário incluir os efeitos da anisotropia na correção da irradiação difusa. Os modelos de Hay (1979) e Perez e outros (1987) superestimam os valores em todos os meses do ano, com valores máximos em torno de $0,4 \mathrm{MJ} / \mathrm{m}^{2}$ durante 0 inverno, sendo os valores de EM ligeiramente menores para o modelo de Hay (1979).

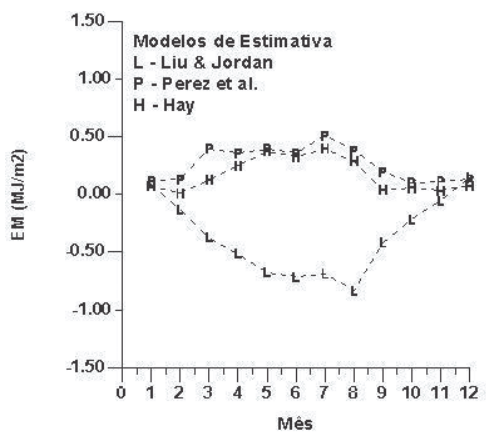

Figura 3 - EM mensal para a irradiação total diária estimada com os modelos de Liv e Jordan (1963), Hay (1979) e Perez e outros (1987).

Figure 3 - Daily total irradiation monthly ME estimated with the models of Liv and Jordan (1963), Hay (1979) and Perez and others (1987).
Os valores de REQM, obtidos mensalmente com os modelos de estimativa da irradiação total diária, estão mostrados na Figura 4. Observa-se que para todos os modelos, os REQM são maiores durante outono e inverno do que durante a primavera e verão, e o modelo de Hay tem os menores valores de REQM em todos os meses, sendo este o melhor modelo para estimar a irradiação total diária na superfície inclinada em Botucatu - SP, por apresentar os menores erros-médios e menores valores de dispersão.

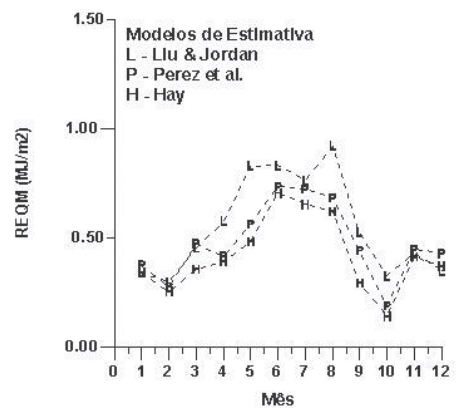

Figura 4 - REQM mensal para a irradiação total diária estimada com os modelos de Liv e Jordan (1963), Hay (1979) e Perez e outros (1987).

Figure 4 - Daily total irradiation monthly RMSE calculated with the models Liu and Jordan (1963), Hay (1979) and Perez and others (1987).

Na Figura 5 estão mostrados os EM resultantes da validação mensal da irradiação total diária na superfície inclinada utilizando os modelos de estimativa de Liv e Jordan (1963), Perez e Seals (1987) e Hay (1979), modificados por $K_{d}=f\left(K_{t}\right)$.

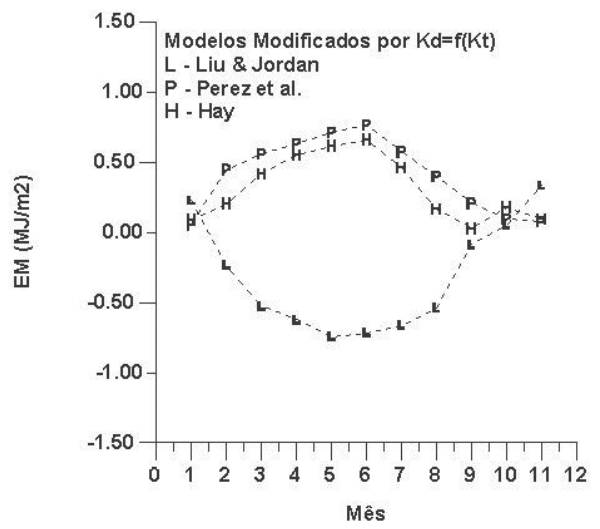

Figura 5 - EM mensal para a irradiação total diária validada com os modelos de Liv e Jordan (1963), Hay (1979) e Perez e outros (1987), modificados por $K_{d}$.

Figure 5 - Daily total irradiation monthly ME validated with the models Liv and Jordan (1963), Hay (1979) and Perez and others (1987), using a modified $K_{d}$. 
Na análise dos erros verifica-se que, novamente, o modelo de Liv e Jordan subestima os valores da irradiação, principalmente durante 0 outono e 0 inverno, enquanto que os outros modelos superestimam os valores em todos os meses do ano. Quando os erros são comparados com os obtidos nos modelos de estimativa originais (Figura 4), verificase que os valores de EM permanecem quase inalterados para 0 modelo de Liv e Jordan (1963) durante os meses do inverno e outono e aumentam no começo do verão e final da primavera. 0 modelo de Hay (1979), apresenta pouco aumento dos EM durante a primavera e verõo, e atinge um máximo de $0,5 \mathrm{MJ} / \mathrm{m}^{2}$ durante 0 inverno, 0 mesmo comportamento é observado para o modelo de Perez e outros (1987), onde durante 0 inverno o modelo apresenta erros da ordem de $0,7 \mathrm{MJ} / \mathrm{m}^{2}$.

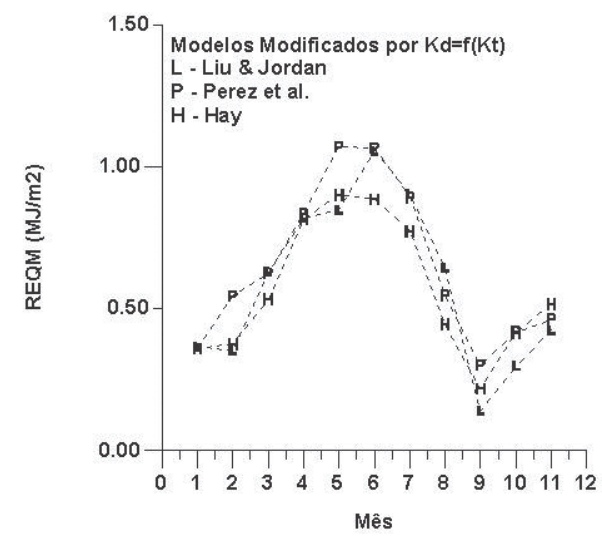

Figura 6 - REQM mensal para a irradiação total diária validada com os modelos de Liv e Jordan (1963), Hay (1979) e Perez e outros (1987), modificados por $K_{\text {d }}$.

Figure 6 - Daily total irradiation monthly RMSE validated with the models of Liv and Jordan (1963), Hay (1979) and Perez and others (1987), using a modified $K_{d}$.

Na Figura 6 estão mostrados os REQM resultantes da validação mensal da irradiação total diária na superfície inclinada utilizando os modelos de estimativa de Liv e Jordan (1963), Perez e outros (1987). e Hay (1979), modificados por $K_{d}=f\left(K_{t}\right)$. Na análise, verifica-se que os REQM seguem o mesmo comportamento dos EM, permanecem quase que inalterados para 0 modelo de Liv e Jordan (1963), aumentam em todos os meses para o modelo de Perez e outros (1987) principalmente durante 0 outono e inverno atingindo um pico máximo no mês de julho em torno de $1,2 \mathrm{MJ} / \mathrm{m}^{2}$, e têm pouco aumento durante a primavera e verõo, o modelo de Hay (1979) também apresenta aumento nos valores da dispersão com pico em torno de $0,8 \mathrm{MJ} / \mathrm{m}^{2}$ durante 0 outono $\mathrm{e}$ inverno e ficam praticamente inalterados durante a primavera $\mathrm{e}$ verõo.

Quando os modelos são modificados através de $K_{d^{\prime}}$, excetuando-se 0 modelo de Liv e Jordan (1963), onde os erros permanecem praticamente inalerados, tanto os EM quanto os REQM aumentam para todos os outros modelos, sendo extremamente elevados durante os meses de inverno e outono.

Na Figura 7 estão mostrados os EM resultante da validação mensal da irradiação total diária na superfície inclinada utilizando os modelos de estimativa de Liv e Jordan (1963), Perez e outros (1987), Hay (1979), modificados por $K_{B H}=f\left(K_{t}\right)$ Na análise dos erros verifica-se que 0 modelo de Liv e Jordan continua subestimando os valores da irradiação total, principalmente durante 0 outono e inverno, e superestima em alguns meses da primavera e verão, no entanto são encontradas melhorias significativas, tornando esse modelo compatível com os outros modelos modificados de estimativa da irradiação total diária na superfície inclinada. Os modelos de Perez e outros (1987) e Hay (1979) superestimam a irradiação total em todos os meses do ano, mas nesse caso, os EM são similares e praticamente iguais aos obtidos com os modelos originais, sendo novamente o modelo de Hay (1979) o que apresenta os menores erros.

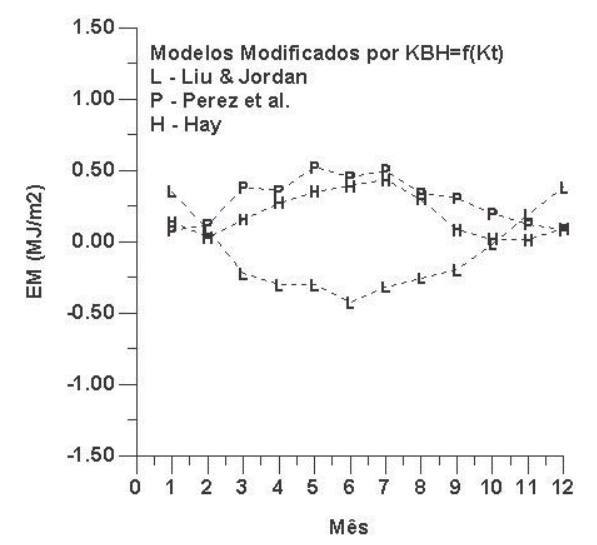

Figura 7 - EM mensal para a irradiação total diária validada com os modelos de Liv e Jordan (1963), Hay (1979) e Perez e outros (1987), modificados por $K_{B H}$

Figure 7 - Daily total irradiation monthly ME validated with the models of Liv and Jordan (1963), Hay (1979) amd Perez and others (1987), using a $\operatorname{modified} K_{B H}$

Na Figura 8 estão mostrados os REQM resultantes da validação mensal da irradiação total diária na superfície inclinada utilizando os modelos de estimativa de Liv e Jordan (1963), Perez e outros (1987), Hay (1979), modificados por $K_{B H}=f\left(K_{t}\right)$ Na análise dos REQM verificase que 0 modelo de Liv e Jordan apresenta valores de dispersão menores do que os obtidos com o modelo original tornando o modelo modificado compatível com o de Hay tendo praticamente os mesmos valores de dispersão e com menores valores do que o modelo de Perez e outros. 


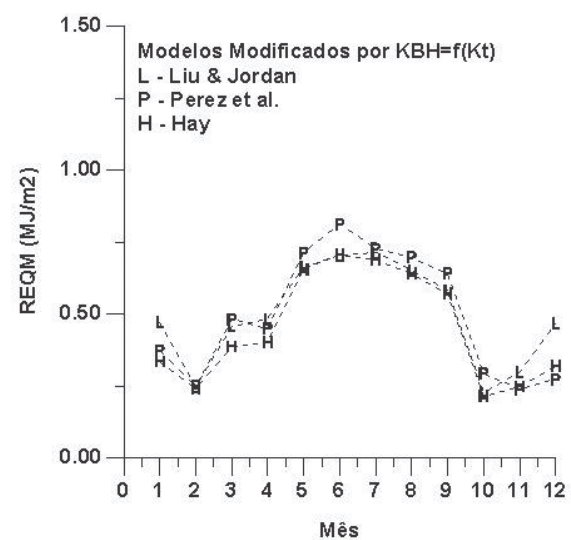

Figura 8 - REQM mensal para a irradiação total diária validada com os modelos de Liv e Jordan (1963), Hay (1979) e Perez e outros (1987), modificados por $K_{B H}$

Figure 8 - Daily total irradiation monthly RMSE validated with the models Liv and Jordan (1963), Hay (1979) and Perez and others (1987), using a modified $K_{B H}$.

\section{CONCLUSÕES}

Os resultados obtidos com os modelos demonstram que o modelo de Liv e Jordan (1963) subestima a irradiação total principalmente durante 0 outono e inverno, enquanto que os modelos de Perez e outros (1987) e Hay (1979) superestimam em todos os meses do ano.

Para os modelos modificados, a comparação entre os erros com as duas modificaçoces feitas, mostra que os erros têm um aumento considerável para todos os modelos, exceto para o de Liv e Jordan (1963), quando a modificação e feita através de $K_{d}=f\left(K_{t}\right)$, demonstrando que quando a irradiação difusa é estimada através de regressão linear múltipla com um polinômio de $4^{0}$ grau, e usada nos modelos modificados, não produz resultados satisfatórios quando se utiliza um conjunto de dados diferente daqueles nos quais ela foi obtida.

Para os modelos modificados através de $K_{B H}=f\left(K_{t}\right)$, os erros aumentam pouco em comparação com os obtidos com os modelos originais, obtendo-se melhorias significativas para 0 modelo de Liv e Jordan tornando-o compatível com os outros modelos, esse resultado demonstra que quando a irradiação direta na horizontal é estimada através de regressão linear com um polinômio de $1^{0}$ grau, e usada nos modelos modificados, produz resultados satisfatórios quando se utiliza um conjunto de dados diferente daqueles em que ela foi obtida, e podem ser usados para estimar a irradiação total em uma superfície inclinada com razoável precisão, sendo o modelo de Hay ligeiramente superior aos outros e o mais indicado para ser utilizado na estimativa da irradiação total em Botucatu-SP.

\section{Agradecimentos}

Os autores agradecem a FAPESP pelo apoio financeiro que possibilitou a montagem da Estaçãa de Radiometria Solar da UNESP em Botucatu-SP.

\section{REFERÊNCIAS}

BEHR, H.D. Solar radiation on tilted south oriented surface validation of transfer-models. Solar Energy, [S.I.], v. 61, n. 6, p. 399-413, 1997. BUGLER, J.W. The determination of hourly insolation on an inclined plane using a diffuse irradiance model based on hourly measured global horizontal insolation. Solar Energy, [S.I.], v. 19, p. 477-91, 1977. FEUERMANN, D.; ZEMEL, A. Validation of models for global irradiance on inclined planes. Solar Energy, [S.I.], v. 48, n. 1, p. 59-66, 1992. ERBS, D. G.; KLEIN, S. A.; DUFFIE, J. A. Estimation of the diffuse radiation fraction for hourly, daily and monthly-average global radiation. Solar Energy, [S.I.], v. 28, p. 293-302, 1982.

GUEYMARD, C. An anisotropic solar irradiance model for tilted surfaces and its comparison with selected engineering algorithms. Solar Energy. v. 38 ก. 5 p. $367-386.1987$.

. Mathematically integrable parameterization of clear-sky beam and global irradiances and its use in daily irradiation applications. Solar Energy, [S.I.], v. 50, n. 2, p. 385-97, 1993.

HAY, J. E. Calculation of monthly mean solar radiation for horizontal and inclined surfaces. Solar Energy, [S.I.], v. 23, p. 301-07, 1979.

IQBAL, M. An introduction to solar radiation. Canada: Academic Press, 1983.

KLEIN, S.A. Calculation of monthly average insolation on tilted surfaces. Solar Energy, [S.I.], v. 19, p. 325-29, 1977.

KLUCHER, T. M. Evaluation of models to predict insolation on tilted surfaces. Solar Energy, [S.I.], v. 23, p. 111-14, 1979.

LIU, B. Y. H.; JORDAN, R. C. The interrelationship and characteristic distribution of direct, diffuse and total solar radiation. Solar Energy, [S.I.], v. 4, p. 1-19, 1960.

LIU, B. Y. H.; JORDAN, R. C. The long term average performance flat plate solar energy collectors. Solar Energy, [S.I.], v. 7, p. 53-74, 1963. NOTTON, G.; MUSELLI, M.; LOUCHE, A. Two estimation methods for monthly mean hourly total irradiation on tilted surfaces from monthly mean daily horizontal irradiation from solar radiation data of Ajaccio, Corsica. Solar Energy, [S.I.], v. 57, n. 2, p. 141-53, 1996.

OLIVEIRA, A. P. et al. Correlation models of diffuse solar-radiation applied to the city of São Paulo, Brazil. Applied Energy, [S.I.], v. 71, p. 59-73, 2002. 
PEREZ, R.; SEALS, R.; INEICHEN, P.; STEWART, R.; MENICUCCI, D. A new simplified version of the Perez diffuse irradiance model for tilted surfaces. Solar Energy, [s.I.], v. 39, n. 3, p. 221-31, 1987.

RICIERI, R. P. Modelos de estimativas e avaliação dos métodos de medida da radiação solar difusa. 1998. 89f. Tese (Doutorado em Energia na Agricultura)-Faculdade de Ciências Agronômicas, Universidade Estadual Paulista, Botucatu, 1998.

TEMPS, R. C.; COULSON, K. L. Solar radiation incident upon slopes of different orientations. Solar Energy, [S.I.], v. 19, p. 179-84, 1977.

\section{NOTAS SOBRE AUTORES}

Dr. José Scolar é bacharel em Física pela Pontifícia Universidade Católica de São Paulo em 1976. Mestrado em meteorologia pelo Instituto Astronômico e Geofísico da Universidade de São Paulo em 1983. Doutorado pela Faculdade de Ciências Agronômicas da UNESP - Campus de Botucatu em 2003. Atualmente é pesquisador aposentado pelo Instituto de Pesquisas Meteorológica (IPMet-UNESP). Suas atividades concentram-se na investigação dos processos de transporte turbulento na camada limite planetária, e na investigação do comportamento da radiação solar recebidas em superfícies inclinadas.

Dr. Dinival Martins é engenheiro florestal pela Escola Superior da Agricultura " Luiz de Queiroz " - USP, Piracicaba-SP em 1977. Mestrado em Energia Nuclear aplicada à Agricultura pelo centro de Energia Nuclear na Agricultura - USP, Piracicaba em 1981. Doutorado em Solos e Nutrição de Plantas - USP, Piracicaba em 1986. É professor da Faculdade de Ciências Agronômicas da UNESP, Campus de Botucatu-SP onde leciona as disciplinas de graduação: Meteorologia e Climatologia; Climatologia Aplicada e auxilia na disciplina de Climatologia Agrícola. No curso de Pós-graduaç̧ão em Energia na Agricultura, é responsável pelas disciplinas: Termodinâmica Atmosférica; e Aproveitamento de Energia Solar. Desenvolve pesquisas com Fontes Alternativas de Energia Natural.

Dr. João Francisco Escobedo é licenciado em Física pela UNESP em 1974 e doutorado em Física pelo Instituto de Física de São Carlos/ USP em 1987. Atualmente é professor adjunto pelo Departamento de Recursos Naturais da UNESP-Botucatu e desenvolve atividades acadêmicas para os cursos de graduação em agronomia, lecionando a disciplina Climatologia Agrícola, e na Pós-graduação é responsável pelas disciplinas Radiação Solar, Técnicas e Medidas de Parâmetros Agrometeorológicos e Processos Térmicos da Radiação Solar para as áreas Energia na Agricultura e Irrigação e Drenagem. Desenvolve pesquisas na área de radiação solar com linhas direcionadas para Instrumentaçã̃o, Medidas, Modelos e Conversão Energética. 